\title{
Visualization of Heat Transfer Using Projector- Based Spatial Augmented Reality
}

Karljohan Lundin Palmerius and Konrad Schönborn

\section{Linköping University Post Print}

\section{Tweet}

N.B.: When citing this work, cite the original article.

Original Publication:

Karljohan Lundin Palmerius and Konrad Schönborn, Visualization of Heat Transfer Using Projector-Based Spatial Augmented Reality, 2016, Augmented Reality, Virtual Reality, and Computer Graphics, pp 407-417.

http://dx.doi.org/10.1007/978-3-319-40621-3_29

Copyright: Springer Verlag (Germany)

http://www.springerlink.com/?MUD=MP

Postprint available at: Linköping University Electronic Press

http://urn.kb.se/resolve?urn=urn:nbn:se:liu:diva-129415 


\title{
Visualization of Heat Transfer using Projector-based Spatial Augmented Reality
}

\author{
Karljohan Lundin Palmerius and Konrad Schönborn \\ Linköping University, Sweden \\ karljohan.lundin.palmerius@liu.se
}

\begin{abstract}
Thermal imaging cameras, commonly used in application areas such as building inspection and night vision, have recently also been introduced as pedagogical tools for helping students visualize, interrogate and interpret notoriously challenging thermal concepts. In this paper we present a system for Spatial Augmented Reality that automatically projects thermal data onto objects. Instead of having a learner physically direct a hand-held camera toward an object of interest, and then view the display screen, a group of participants can gather around the display system and directly see and manipulate the thermal profile projected onto physical objects. The system combines a thermal camera that captures the thermal data, a depth camera that realigns the data with the objects, and a projector that projects the data back. We also apply a colour scale tailored for room temperature experiments.
\end{abstract}

Keywords: Spatial Augmented Reality, Thermal Imaging, Real-time Projection Mapping, Science Education

\section{Introduction}

Thermal imaging cameras, also referred to as infrared thermography (IRT) cameras, are utilised to detect mid- and longwave infrared radiation emitted from objects. The resulting images are rendered in various pseudo-colour scales to enable human perception of the warmest and coolest parts of viewed surfaces. Thermography is used in multiple applications that include building inspection, automotive night vision, search and rescue, and medical diagnosis. More recently, IRT cameras have been empirically investigated as pedagogical tools for helping students visualize, interrogate and interpret notoriously challenging thermal concepts[7]. Herein, the use of IRT cameras for learning offers a form of educational technology that makes otherwise invisible processes visible[14,12]. In this regard, we have used IRT cameras in science education contexts[7,6] for direct macroscopic visualization of thermal concepts related to heat transfer (e.g. conduction and thermal insulation), energy transformations (e.g. kinetic to thermal), and other dissipative processes (e.g. dry friction).

To date, our investigations have involved students viewing the thermal camera display of the hand-held camera while performing different tasks to interpret thermal phenomena, or where the image feed is projected onto a screen. As an alternative approach, we hypothesise that an Augmented Reality (AR) system could offer a more natural visual feedback and intuitive learning experience for interpreting abstract science concepts (e.g. [13]). In this manner, instead of having to physically direct a hand-held 
camera toward an object of interest, and then view the display screen as the graphical overlay of the thermal world, what if the thermal image could be projected directly onto the object surface? In addition, we are of the view that an augmented display may also open up opportunities for novel collaborative learning spaces[8], where more than one participant can simultaneously manipulate thermal phenomena during physical interaction with the AR system.

In this paper we present a system for Spatial Augmented Reality (SAR), displaying thermal data onto real objects, as a part of our ongoing efforts on exploring the use of thermal imaging in education. The main contributions of the paper are

- the presentation of a technique for displaying thermal data onto real objects through real-time projection mapping,

- the presentation of a complete hardware and software concept for a fully 3D thermal projection system, including mounting and discussion of shadow concepts, and

- a colour scheme specially designed for the projection of thermal data for heatrelated experiments at $\pm 10^{\circ} \mathrm{C}$ of room temperature.

\section{Related Work}

Spatial Augmented Reality (SAR) was first introduced by Raskar et al. in [11], as an approach to provide Augmented Reality without introducing head-mounted display systems. The use of structured light projection to extract surface geometries was cited as a technique to compensate for the shape of the projection surface. Today this is encapsulated as consumer-available depth cameras. Two of the authors later published a book on the subject[2]. A more recent example of projector-based SAR is the work by Benko et al.[1], in which multiple projectors are used to turn all surfaces in a room into a 3D display system.

When it comes to thermal imaging, there has been some work on the use of projectorbased Augmented Reality, however to the authors current knowledge, nothing has yet been published in the scientific literature. For example, Ken Kawamoto presented in both a blog and an online video what he terms a ThermalTable[9]. He used a FLIR $\mathrm{C} 2$ camera to record thermal data from a table that were then projected back using a projector. The camera and the projector are aligned so that the recorded thermal profile is projected back to its origin, at least on the table surface. For objects above the surface, the projection becomes increasingly misaligned with increasing distance from the surface.

Gladyszewski et al. posted a video[5] showing their "thermal video projection system", initially part of the choreographic project Corps Noir. This system uses a colocated thermal camera, video projector and video camera to create a live video feed that shows thermal information projected onto the bodies of dancers. Since the three devices are co-located their different views can be adjusted to align without taking the shape of the projection surface into account. In continuation of that work, Gladyszewski recently posted a new video of a related artwork[4]. This work is similar to that of Kawamoto, and the thermal image is instead projected back onto a table-top clay surface. 
While the former aesthetically attractive examples certainly represent the many possible applications of projector-based thermal AR, the challenge still remains of automatically aligning any $3 \mathrm{D}$ object viewed from any angle. Our work presented here aims to use depth information to create a dynamically updated projection mapping of the thermal information onto any object placed on, or held above the table surface. In turn, such a solution will make the concept more accessible for education purposes.

\section{Display System}

Our projector-based spatial AR display system for thermal data uses a thermal camera to capture thermal data in a region above a small table surface, and a data projector to project those data back to the recorded object. The theoretical optimum is to co-locate the thermal camera and the projector, so that the projection can be perfectly aligned with the recorded data. However, with the current hardware available for this project we also faced the challenge of solving a more complicated placement. This is further explained below.

To obtain correct alignment between the recorded thermal data and the projection, regardless of the presented geometry, we use a depth camera that captures the geometry and enables dynamic, real-time projection mapping[11]. Thus, our system consists of a rig with three imaging devices: a thermal camera, a depth camera and a projector, see Fig. 1.

\subsection{Projector}

The purpose of the projector is to augment objects with visual thermal information by projecting colours onto them as part of a natural educational environment. The most important aspects of the projector are colour reproduction and static contrast. Since the projection area is smaller compared to other applications, brightness is of less importance.

We selected an Epson EB-1940W, a three-panel LCD projector, for our prototype. Since it is recommended not to be mounted vertically, like most projectors of that type, we use a mirror to redirect the projection towards the table surface, see Fig. 1(d).

\subsection{Depth Camera}

The depth camera used in our system is a Microsoft Kinect Version 2, which is an active camera based on structured light in the N-IR spectrum. It is factory calibrated but requires a minimum distance of approximately $400 \mathrm{~mm}$ to the nearest objects. Therefore, to allow users of the system to hold objects above the table surface, we mounted the depth camera at a height of about $750 \mathrm{~mm}$.

We have designed and 3D printed holders to mount the camera as close as possible to the origin of the projection, see Fig. 1(d). 


\subsection{Thermal Camera}

The thermal camera included in our display system is a FLIR E4, an entry level device with $80 \times 60$ pixels sensor resolution, which is much lower than a conventional camera. The camera provides live video streaming via USB Video Class (UVC) standard, and includes both thermal scale legend and other information. These do not represent thermal data and should thus be masked out for a better user experience.

Due to the low resolution, we cannot afford to record a large area of the table and since the camera has a fixed lens, this means that the camera has to be mounted close to the table surface. This means that the thermal camera has to be mounted to the side, to avoid it occluding the two other imaging devices. We decided on a distance of $350 \mathrm{~mm}$, resulting in a pixel size of $\sim 4 \mathrm{~mm}$ over a surface of 300 by $250 \mathrm{~mm}$ after masking out non-thermal video features.

The camera is designed for hand-held use and therefore has no mount. To fix it to the display system we again designed and 3D printed appropriate holders, see Fig. 1(b).

\subsection{Projection Calibration}

There are three different imaging devices in this system that need to be calibrated and co-registered to the same coordinate system: the projector, the depth camera and the thermal camera. All three devices have their own special features making it a non-trivial task to find the correspondances necessary for the calibration. The depth camera only detects depth differences, the thermal camera requires a target with a higher or lower temperature than the surroundings, and the projector cannot detect anything.

The depth camera defines the coordinates of the system. Thus, the depth camera/projector and the depth camera/thermal camera registration needs to be estimated. We did this by first finding corresponding points both in 3D on the depth camera and in $2 \mathrm{D}$ on the projector and thermal camera, respectively. There are ways to automatically find correspondences between a projector and a calibration target (see e.g. [10]). However, since the thermal camera requires separate solution anyway, we chose to use a manual method for the projector: we manually move graphics projected by the projector to align it with a target visible to the depth camera, see Fig. 1(c).

The target we use consists of a soaked paper sheet, placed on top of a plastic surface for stability. The paper cools due to evaporation and a hole in the surface can be detected by both the depth camera and the thermal camera, while it is also easy to align a projected pattern to it.

The manually collected correspondences are then used in OpenCV's calibrateCamera function to find the intrinsics and extrinsics of the projector and the thermal camera, respectively, relative to the $3 \mathrm{D}$ space defined by the depth camera.

\section{Colour Scheme}

The primary purpose of the prototype presented here is to visualize thermal processes occuring near room temperature. So, the most important feature is to visualize where the temperature diverges from ambient room temperature. For this purpose we have 


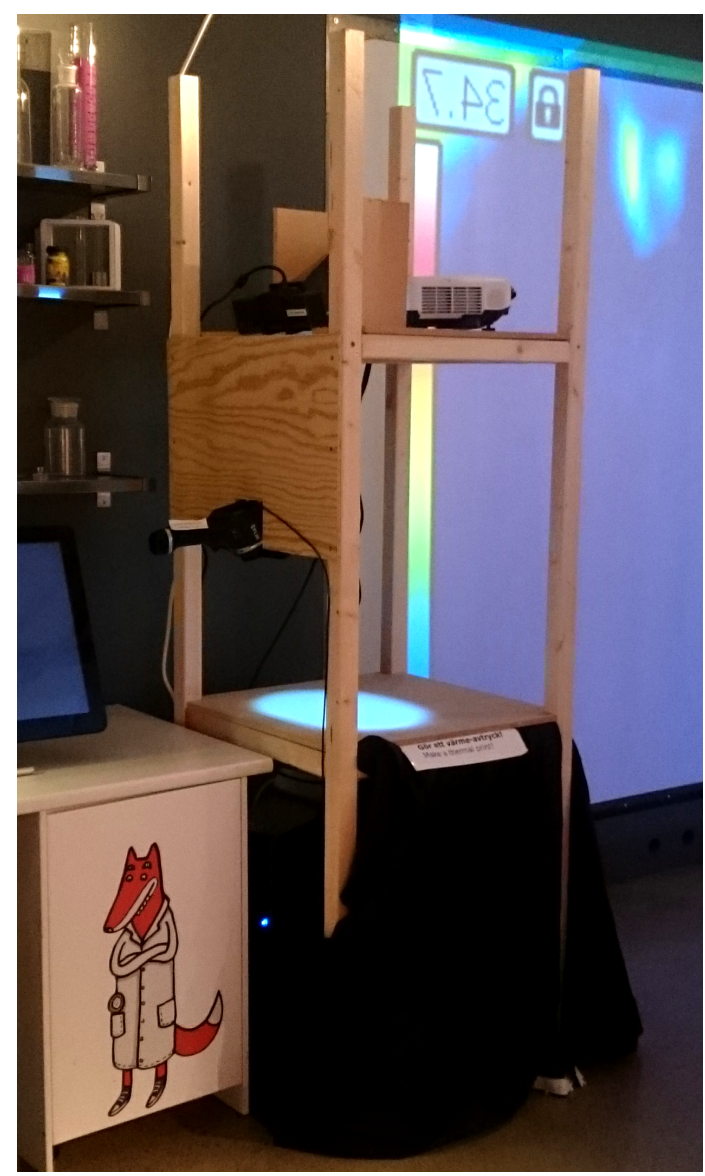

(a) The full rig with all equipment mounted, placed in a public exhibition at a digital science centre.

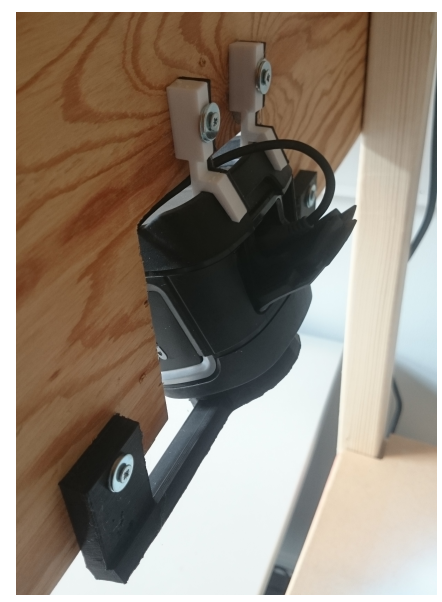

(b) The mounted FLIR E4.

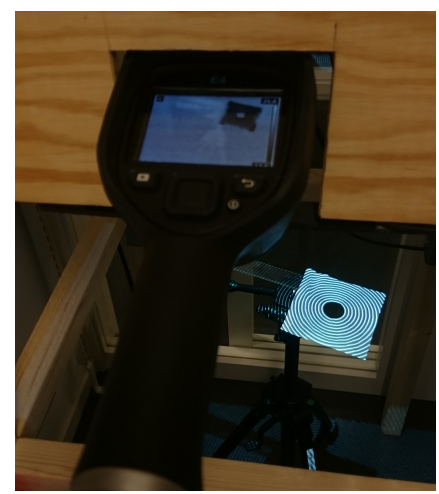

(c) The calibration target.

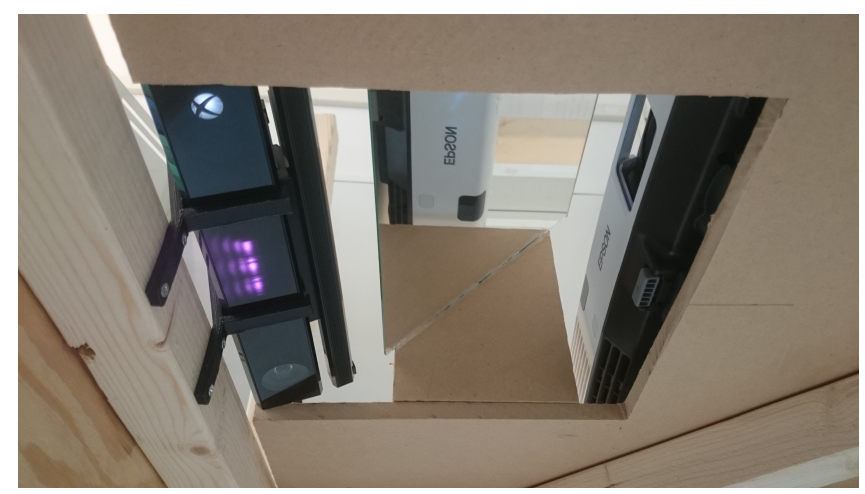

(d) The Kinect and the projector with accompanying mirror.

Fig. 1: Photographs of the thermal SAR display system, and comprising components. 
specially designed a colour scale that represents room temperature as black, so that the projector leaves those objects unaugmented.

To obtain high colour contrasts while allowing for a high dynamic range, we have selected four colours to represent temperatures $10^{\circ} \mathrm{C}$ below, $5^{\circ} \mathrm{C}$ below, $5^{\circ} \mathrm{C}$ above and $10^{\circ} \mathrm{C}$ above room temperature, respectively, see Fig. 2. This temperature range covers educational thermal experiments that we have conducted to date[7].

The FLIR E4 uses a dynamic scale. To realize this colour scale, the video feed data have to be converted. First, the camera is set to display a gray scale representation of the thermal data. The video feed contains a numeric scale showing the range of the data, which we read off using OCR. Every gray scale thermal pixel, $t_{i, j}$ in the range 0-255, can then be converted to its corresponding absolute temperature value, $T_{i, j}$, by

$$
T_{i, j}=\frac{t_{i, j}}{255}\left(T_{\max }-T_{\min }\right)+T_{\min }
$$

where $T_{\min }$ and $T_{\max }$ are the minimum and maximum temperature in the scale, respectively. It is then straightforward to apply the colour scale described above.

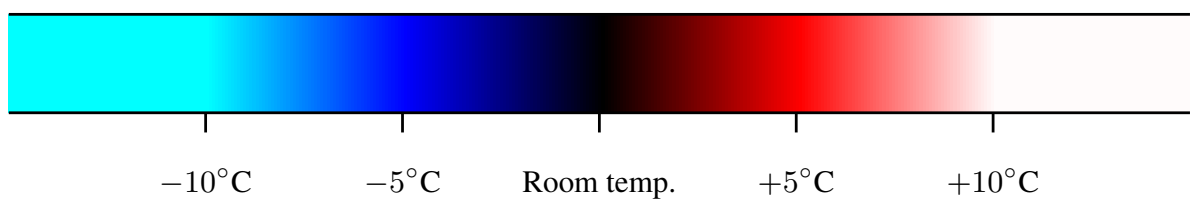

Fig. 2: Our colour scale designed for projector-based augmentation with thermal data. The scale is centered on room temperature, which is rendered black, and ranges from $10^{\circ} \mathrm{C}$ below to $10^{\circ} \mathrm{C}$ above room temparature, respectively, through cyan-blue-blackred-white. This means that the projector only augments objects that are affected by heat transfer outside of room temperature.

\section{Software Implementation}

The software that generates the projector output is implemented with OpenSceneGraph (OSG), a high performance, OpenGL-based scenegraph library. It uses two main components encoded into scenegraph nodes: the DepthMesh node, providing a 3D mesh that leads to correct projection mapping, and the ThermalTexture node, which maps the thermal imaging onto that mesh as a texture. The software also applies special lighting effects to remove artifacts, which is discussed further in section 6.

\subsection{The ThermalTexture Node}

The ThermalTexture node reads off the video feed from the thermal camera, applies the colour scale described above and encodes the image as an OSG texture. The FLIR E4 communicates via USB as a UVC device where we use OpenCV to capture for cross platform support. The temperature scale, needed for the colour scale as described above, is extracted from the video feed using the Tesseract OCR library. 


\subsection{The DepthMesh Node}

Our DepthMesh node uses libfreenect2[3] to read off depth data from the Kinect camera, for cross platform support. Each pixel, $(i, j)$ with depth $d_{i, j}$, in the depth image is then mapped to a $3 \mathrm{D}$ position, $\boldsymbol{p}$, through

$$
\begin{aligned}
& p_{x}=d_{i, j} \frac{i-W / 2}{f_{x}} \\
& p_{y}=d_{i, j} \frac{j-H / 2}{f_{y}} \\
& p_{z}=-d_{i, j}
\end{aligned}
$$

where $W$ and $H$ are the image width and height, respectively, and $f_{x}$ and $f_{y}$ are the camera's focal length in pixels in $\mathrm{x}$ and $\mathrm{y}$, respectively, taken from the camera intrinsics obtained in the calibration step described above. For each square of four pixels, if at least three of them contain valid depth data, then this square can be triangulated into a patch. When all sets of $2 \times 2$ pixels have been triangulated, we have a mesh representing the depth data from the camera, which is encoded as a OSG geometry.

It is possible to use hole filling algorithms to compensate for missing data from the depth camera. However, our experience with the current system is that incorrect data, which cannot be corrected this way, is more common than missing data.

The ThermalTexture, described above, is applied to colour the DepthMesh geometry. The correct texture coordinates, $(u, v)$, for the thermal data on the mesh, are calculated using the thermal camera's intrincis and extrinsic matrices,

$$
w\left(\begin{array}{c}
u \\
v \\
1
\end{array}\right)=M_{\text {intr }} M_{\text {extr }}\left(\begin{array}{c}
p_{x} \\
p_{y} \\
p_{z} \\
1
\end{array}\right)
$$

where $M_{\text {extr }}$ is the $4 \times 4$ camera extrinsics matrix and $M_{\text {intr }}$ is the $3 \times 4$ camera intrinsics matrix.

\section{Shadows in the System}

Any 3D object placed in the system will cast shadows. The most intuitive shadow is that appearing behind the object from the projector's point of view, because of the occlusion of the projector light. However, the two cameras will also generate shadows. First, the depth camera cannot see behind a solid object and there will therefore be a "shadow", actually a hole in the 3D mesh, behind the object from the depth camera's point of view.

Second, the thermal camera cannot detect thermal data behind non-transparent objects. However, the texture coordinates that map the thermal data onto the mesh are directly calculated from the 3D position of the mesh and therefore do not take this into account. Thus, the thermal data of an object is cast onto background surfaces as a ghost image of that object. This projection does not represent the thermal signature of that surface and needs to be digitally removed. 
Fortunately, the OpenSceneGraph library used for the software has support for realtime shadow rendering. We deploy the ShadowMap functionality, which implements the shadow map algorithm, to cast shadows configured as black. This algorithm supports self-shadowing, which is necessary since it is the part of the mesh that represents the object in front of the camera that should cast shadows onto another part of the same mesh. The result is black shadows behind objects with respect to the thermal camera, which removes most of the ghost image that was otherwise fully visible, see Fig. 3. The shadow and ghost image will never match perfectly due to discretization and also, in particular, due to the low resolution of the thermal camera.

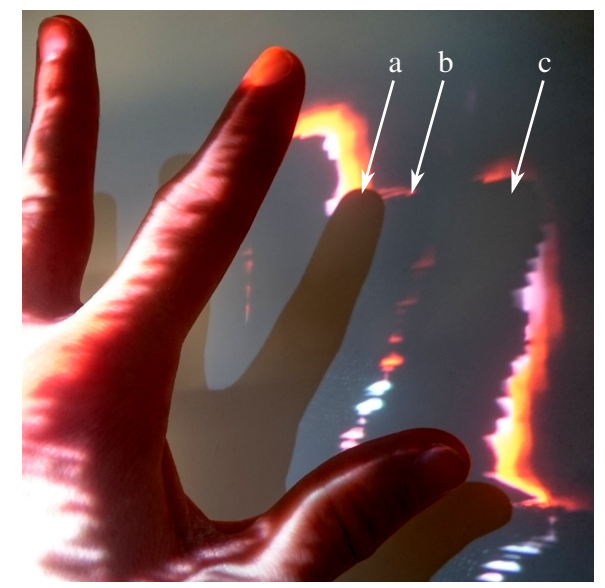

Fig. 3: The three shadows cast behind an object in our display system, from the point of view of a) the projector, b) the depth camera and c) the thermal camera.

\section{Results}

This work has resulted in a thermal Spatial Augmented Reality display system that superimposes thermal information onto objects, using a colour scheme that focusses on visualizing heat phenomena near room temperature range. Objects placed on the table or held above are augmented only on surfaces facing all imaging devices and within all their respective fields of view. This is not a large volume, partly because of the placement of the thermal camera, but is still sufficient for exploration and basic experimentation, see Fig. 4.

Non-reflective, non-transparent objects work best with both the thermal camera and the depth camera. All resonably bright objects adequately reflect the colours displayed by the projector, but white or bright gray objects are augmented more accurately, since this system does not apply any surface colour compensation[2].

The projector produces hot air and early experiments indicate that it is important that the air stream is directed away from the rig to avoid it heating the table surface. 
Our preliminary observations indicate that the AR system allows for real-time visualization of various heat phenomena. For example, we have used the system to conduct tasks envisioned for public (e.g. science center) and formal (e.g. school) science learning contexts. These include the thermal visualization of the surface of one's hand, heat transfer from one's palm to the wooden table surface, liquid water at different temperatures (and the subsequent mixing thereof), latent heat of evaporation, and rubbing an eraser across a rough surface.

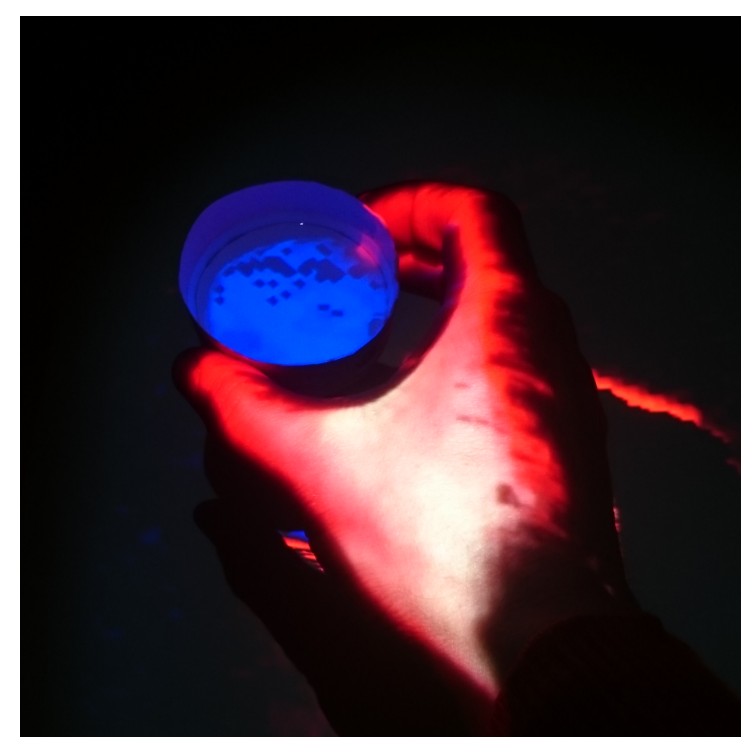

Fig. 4: The AR system projecting real-time thermal data onto a hand holding a cup of water. Warmer areas on the surface of the hand are displayed in red and white, relative to the cooler water surface in blue. The surroundings are at room temperature (black).

\section{Conclusions and Future Work}

We conclude that the presented hardware combination-Kinect depth camera, FLIR E4 thermal camera and Epson EB-1940W projector-used with the described software constellation results in thermal data intuitively presented on the objects in the Spatial Augmented Reality system. The proposed colour scale effectively highlights objects or regions with temperatures that diverge from room temperature. Multiple experiments can be carried out to demonstrate the educational implications of the system for visualizing thermal concepts. Future work will explore the role of the AR system in communication, learning and teaching in this regard.

Whether or not the proposed colour scale is inuitive with respect to what is warmer and what is cooler is yet to be formally examined and shall constitute a forthcoming perception study. 


\section{Acknowledgments}

We thank Anna-Karin Lindblom, Product Manager at FLIR Systems AB, for supporting this work. We also thank Dr. Fredrik Jeppsson, Linköping University, for the loan of a FLIR E4 camera, and Dr. Jesper Haglund for useful discussions.

\section{References}

1. Benko, H., Wilson, A.D., Zannier, F.: Dyadic projected spatial augmented reality. In: in Proceedings of The ACM Symposium on User Interface Software and Technology (UIST). pp. 645-655 (2014)

2. Bimber, O., Raskar, R.: Spatial Augmented Reality - Merging Real and Virtual Worlds. A K Peters/CRC Press (2005)

3. Blake, J., Kerl, C., Echtler, F., Xiang, L.: libfreenect2: Open-source library for kinect v2 depth camera. Zenodo (January 2016), http: / / dx. doi .org/10.5281/ zenodo. 45314

4. Gladyszewski, S.: Argile et lumière - clay and light. https://vimeo.com/ $152905116(2016)$

5. Gladyszewski, S., Burton, A., Ricard, J., Étienne Grenier: Live thermal video projection system. https: / /vimeo.com/60292952 (2013), accessed 2016-02-12

6. Haglund, J., Jeppsson, F., Hedberg, D., Schönborn, K.J.: Students' framing of laboratory exercises using infrared cameras. Phys. Rev. ST Phys. Educ. Res. 11(2) (2015)

7. Haglund, J., Jeppsson, F., Melander, E., Pendrill, A.M., Xie, C., Schönborn, K.J.: Infrared cameras in science education. Infrared Physics \& Technology 75, 150-152 (March 2016)

8. Johnson-Glenberg, M.C., Birchfield, D.A., Tolentino, L., Koziupa, T.: Collaborative embodied learning in mixed reality motion-capture environments: Two science studies. Journal of Educational Psychology (2014)

9. Kawamoto, K.: Thermaltable. http://kenkawamoto-works.tumblr.com/post/ 106298696083 /thermaltable-2014-dec-link-using-flir-one-an (December 2014)

10. Lee, J., Dietz, P.H., Maynes-Aminzade, D., Raskar, R., Hudson, S.: Automatic projector calibration with xembedded light sensors. In: Proceedings of the ACM Symposium on User Interface Software and Technology (UIST) (October 2004)

11. Raskar, R., Welch, G., Fuchs, H.: Spatial augmented reality. In: Proceedings of The IEEE International Workshop on Augmented Reality (1998)

12. Vollmer, M., Möllmann, K.P., Pinno, F., Karstädt, D.: There is more to see than eyes can detect - visualization of energy transfer processes and the laws of radiation for physics education. Phys. Teach 39(6), 371-376 (2001)

13. Wu, H.K., Lee, S.W.Y., Chang, H.Y., Liang, J.C.: Current status, opportunities and challenges of augmented reality in education. Computers \& Education 62, 41-49 (2013)

14. Xie, C., Hazzard, E.: Infrared imaging for inquiry-based learning. Phys. Teach. 49(6), 368$372(2011)$ 